\title{
Temperature fluctuations in the unburned mixture: Indirect visualisation based on LIF and numerical simulations
}

\author{
R. SchießI, A. Schubert and U. Maas \\ Institut für Technische Thermodynamik, Universität Karlsruhe (TH)
}

Copyright $\odot 2006$ SAE International

\begin{abstract}
We apply a method for the visualization and semiquantitative estimation of small spatial temperature fluctuations in internal combustion engines with premixed loads. It is based on laser-induced fluorescence (LIF) of formaldehyde $\left(\mathrm{CH}_{2} \mathrm{O}\right)$, which is formed in the unburned gas near the end of the compression stroke. The chemical reactions leading to formaldehyde formation during the phase before auto-ignition are strongly temperaturedependent. The concentration of $\mathrm{CH}_{2} \mathrm{O}$ therefore acts as a natural, very sensitive tag for local gas temperature variations. A correlation between temperature fluctuation and formaldehyde concentration fluctuation is assessed by using numerical simulations involving a detailed treatment of chemical reactions leading to formaldehyde formation in the unburned gas. Formaldehyde is detected in the unburned gas of an optically accessible test $\mathrm{SI}$ engine by laser-induced fluorescence (LIF) along a line. The LIF-signal trace displays pronounced spatial fluctuations, which can not be attributed to inhomogeneities of the fuel/air ratio or to measurement noise, but which must be due to local temperature inhomogeneities in the unburned gas. By combining the observed spatial LIF-signal fluctuations with the computed formaldehyde/temperature correlations, temperature fluctuation amplitudes can be estimated. It is shown that the technique is capable of detecting fluctuations well below $\pm 10 \mathrm{~K}$ before a mean temperature background of $800 \mathrm{~K}$ and higher.
\end{abstract}

The results obtained by applying the method in a simple test engine give evidence that considerable temperature fluctuations exist in the unburned gas, both with respect to their amplitude and to their geometrical size. The information delivered by the method may be important especially in the field of auto-ignition in $\mathrm{HCCl}$-engines, since local temperature inhomogeneities in the unburned gas can be of paramount influence for the subsequent development of self-ignition and combustion.

\section{INTRODUCTION}

The temperature of the unburned gas highly influences the operation of internal combustion engines. This is due to the strong temperature dependence of the chemical reactions by which the fuel is gradually consumed and, eventually, by which self ignition can occur. A too high temperature of the unburned gas can lead to autoignition and engine knock [1]. Throughout the last years, interest in a new engine concept that is based on autoignition has grown considerably. In these $\mathrm{HCCl}$ - (homogeneous charge compression ignition) engines, a premixed load is compressed by the piston until autoignition occurs $[2,3]$. This principle allows engine operation also under lean conditions, allowing an efficient operation with low pollutant formation [4].

A key for the successful operation of these engines is the regulation of the start of self-ignition and the speed of the subsequent combustion. Unlike in spark ignition engines, where ignition and combustion are triggered by the spark, self-ignition occurs as a result of the chemical reactions. These are dependent mainly on temperature, which strongly influences the ignition delay time of a fuel/air mixture [2]. The role of temperature for the autoignition in an SI engine was studied numerically (e.g., [5]). Since a small local variation of temperature (in the range of $10 \mathrm{~K}$ ) can substantially decrease the local ignition delay time [5], it is desirable to obtain very precise information about the temperature field in the unburned gas.

Temperature measurements in the unburned mixture (the endgas of a spark ignited engine) have been performed by researchers using Coherent Anti-Stokes Raman scattering (CARS) [6], or two-line laser-induced fluorescence (LIF) of a tracer [9]. The latter technique has been used for obtaining planar temperature fields while the former is typically employed as a pointmeasurement.

Despite the existence of several dedicated experimental techniques, measuring instantaneous gas temperature fields in unsteady, chemically reacting flows is still a challenging task. Using the established methods (e.g., Rayleigh scattering, or Laser-Induced Fluorescence), it is very hard to reach an accuracy and precision of better than around $20 \mathrm{~K}$ even in environments that are benign for measurements (e.g., free, atmospheric pressure flames). In the combustion chamber of engines, the precision limit can be expected to be somewhat worse. 
Special importance lies on retrieving information about local inhomogeneities of the temperature field. Determining the (spatially averaged) mean of the in-cylinder temperature field with reasonable accuracy might not be too problematic in many cases. However, empirical information about spatial temperature fluctuations (i.e., local deviations from the mean value) is often needed urgently as an input to improve the accuracy of numerical simulations. Also, local temperature inhomogeneities can have an immediate practical significance in engines. For instance, under certain conditions, the combustion in engines based on auto-ignition of premixed loads can be viewed as resulting from a sequence of nearly independent auto-ignition events, occurring at spatially separated sites. The time at which auto-ignition and the subsequent combustion occur at a site in the combustion chamber is determined mainly by the local temperature. In this way, spatial fluctuations of temperature can cause a temporal spread of the auto-ignition events in the combustion chamber, and therefore of overall combustion duration. This can effectively prevent exceedingly high temporal gradients of the cylinder-pressure, as often observed, e.g., in $\mathrm{HCCl}$ engines at high loads. These "beneficial" effects have already been observed and analyzed (see, e.g., [7] and [5]). Studies towards finding the cause of the inhomogeneities (this issue is not addressed in this paper) have also been performed, by other authors, in a $\mathrm{HCCl}$ context [8].

In this work, a method for the semi-quantitative measurement of spatial temperature inhomogeneities in engines is introduced and applied to a simple, optically accessible test engine. The method exploits the strong temperature-dependence of intermediate species concentrations that are formed in the unburned gas before and during ignition in highly temperature dependent chemical reactions.

By numerical simulations of the unburned gas based on detailed treatment of chemistry, it is shown that in the phase shortly before and during auto-ignition, small variations in temperature show up as considerable variations in the concentrations of the intermediate species. These local concentration fluctuations can be detected by laser-induced fluorescence of the intermediate species. From the observed fluctuation amplitude of the LIFsignal, information about the underlying temperature fluctuation can be inferred. After ignition, intermediate species are rapidly consumed, so that their absence indicates the onset of strongly exothermic combustion reactions. We detail the principle of the methodology, explaining the assessment of temperature/species correlations by numerical simulations. These correlations are transformed into correlations between LIF signal- and temperature fluctuations by spectroscopic simulations. After measuring the LIF-signals in an optically accessible test engine, the correlations are used to transform the observed LIF-signal fluctuations into temperature fluctuations. The significance of these temperature fluctuations for engines that operate in self-ignition mode is discussed.

\section{METHODOLOGY}

\section{PRINCIPLE OF THE METHOD}

Temperature measurements are based on the observation of quantities that depend on temperature in a known fashion. In principle, the measurement will be the more precise the stronger the observed quantity depends on temperature.

In the combustion chamber of engines, the formation of certain chemical species by temperature dependent reactions in the fuel/air mixture ranks among the most strongly temperature dependent quantities. This holds especially in the phase shortly prior to or during selfignition. More precisely, the temperature dependence of the velocity of a reaction contains an expression of the form $\exp \left(-E_{\mathrm{a}} /(k T)\right)$, where $E_{\mathrm{a}}$ is an activation energy that is specific for the reaction, and $k$ is Boltzmann's constant. This strong temperature dependence of chemical kinetics is the basis of many important effects in engines, like for example the large sensitivity of autoignition timing with respect to gas temperature.

The temperature fluctuation measurement method described here is based on visualizing chemical species that display such a strong temperature dependence. A species that lends itself to this purpose is formaldehyde $\left(\mathrm{CH}_{2} \mathrm{O}\right)$. Formaldehyde is not present in the unreacted fuel/air mixture (unless it is doped to the fuel), but is formed in strongly temperature dependent reactions during the pre-ignition phase (typically towards the end or shortly after the compression stroke) in engines operating on hydrocarbon fuels. In this phase, it can be present in mole fractions of several thousand ppm. After that, formaldehyde is consumed rapidly by the chemical reactions leading to combustion, and is therefore not present in the burned mixture (or only in negligible amounts). For illustration, Figure 1 shows a qualitative sketch of a typical formaldehyde concentration "history" during the compression and expansion phase in an engine.

Modeling studies and experimental observations [14] indicate that the temperature dependence of formaldehyde concentrations (and also of formaldehyde mole fractions) before and during the ignition phase is extremely strong. The strong temperature dependence, the relatively high mole fractions in the important phase before and during self-ignition, and the ability to be detected by laser-induced fluorescence in engines [12, 14, 23] makes formaldehyde an ideal candidate species for measuring temperature fluctuations in the unburned fuel/air mixture in IC engines. Formaldehyde is, however, not the only species with these properties, but is rather just a member of a whole species class with very similar chemical behaviour before, during and after the ignition phase [13]. It is mainly the fact that many of its spectroscopic properties have been studied comparatively well that makes it the outstanding member of this class. The combined knowledge on spectroscopical and chemical properties substantially alleviates the interpre- 
tation of the LIF signals caused by $\mathrm{CH}_{2} \mathrm{O}$, as shown below. We emphasize that the method does not aim at measuring absolute temperatures. Instead, it is for measuring small spatial temperature fluctuations in the unburned fuel/air mixture, i. e., small deviations of the local temperature from the spatial mean value.

In the pre-ignition and ignition phase, even tiny temperature fluctuations show up as considerable fluctuations in formaldehyde concentrations due to the "amplifying" effect of the strongly temperature dependent chemical formation of formaldehyde. This corresponds to the steepness of the left branch (formation during the compression phase) of the curve in Figure 1.

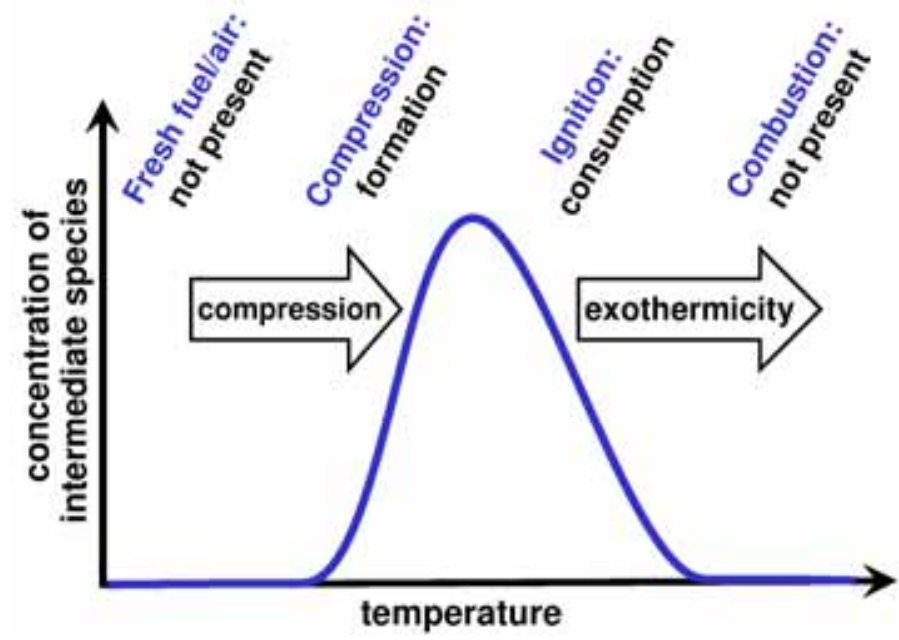

Figure 1 Sketch of the qualitative behaviour of intermediate species concentrations vs. gas temperature in an engine during the compression stroke and the combustion phase in the expansion stroke. The text labels on top indicate the effect of different phases on intermediate species.

In principle, we therefore have a means of indirectly detecting temperature fluctuations by detecting variations of formaldehyde concentrations. Formaldehyde concentrations are, however, not directly observable with laserinduced fluorescence. The quantity that is actually observed in a LIF-experiment is the fluorescence signal caused by formaldehyde. This signal is, of course, related to the concentration of formaldehyde, but it depends also on temperature, due to the thermal population of the laser-excited ground state(s), due to Doppler broadening of spectral lines, etc. It also depends on pressure due to effects like pressure broadening of the absorption features of formaldehyde and quenching. For the same reason, the LIF-signal also is influenced by the concentration of other species that are present in the unburned gas. Means have to be found to infer temperature fluctuations from fluctuations of the LIF-signal.

The measurement therefore consists of three main steps:

1. For the conditions that exist in the unburned gas in an engine, assess correlations between temperature and formaldehyde concentrations. Numerical modelling of the thermo-chemical development of the unburned gas, based on detailed chemical kinetics, is used for this task.

2. Use spectroscopic calculations to estimate the dependence of the formaldehyde LIF-signal on temperature and chemical composition of the unburned gas.

3. Practical application: Measure LIF-signals of formaldehyde in an engine, and check these signals for their spatial fluctuation amplitudes. Using the information from 1. and 2., we then convert these measured fluctuations into temperature fluctuations.

For these steps, it is required to distinguish between the left and the right branch of the curve in Figure 1. The left branch (with a positive slope) corresponds to the formation of intermediate species in the unburned mixture. This is a comparatively slow chemical process, occurring at relatively low temperatures [2]. In this phase, the increase of temperature due to chemical reactions is very small. In fact, the chemical reactions here can even be slightly endothermic. In an engine, this phase is typically realized in the late compression phase, around top dead centre. The right branch (with a negative slope) is due to the consumption of intermediate species after autoignition has started. This is the actual transformation of unburned gas to burned gas, associated with a fast temperature rise due to strongly exothermic chemical reactions. Once the culmination point of the curve in Figure 1 is reached, the gas temperature will start to rise. This in turn, will strongly accelerate chemical reactions, leading to an even faster temperature rise, an so on. The system therefore will progress very quickly towards combustion: Accessing the right branch corresponds to auto-ignition, which, in an engine with a quasi homogeneous, nearstoichiometric, undiluted fuel/air mixture will lead to a very steep rise in the cylinder pressure (typically resulting in engine knock). If the mixture in such an engine does not display self-ignition, it can not have reached the right branch. For the assessment of correlations between species concentration- and temperaturefluctuations, exclusively the left branch of the curve has in our case to be considered.

In the following, we will describe a realisation of the individual steps in detail.

\section{ASSESSMENT OF TEMPERATURE / CONCENTRA- TION CORRELATIONS}

Information about the correlation between temperature and formaldehyde concentrations for conditions that are representative of a simple test engine (see experimental section) was obtained by numerical simulations of the chemical reactions occurring in the cylinder load during an engine cycle. The focus of these simulations is not on modelling an engine with its particular (often not wellknown) set of boundary conditions, but on creating a set of representative simulations that afford insight into the 
correlations between the most important quantities in our approach - the temperature and the concentration of formaldehyde. In the simulations, the cylinder load of an engine is modelled as a homogeneous reactor. To this homogeneous reactor, cylinder pressure traces $p(t)$ as measured in a test engine are applied as a temporal boundary condition to the chemically reacting system. The governing equations for this reactor can be described by the following set of coupled differentialalgebraic equations (DAEs):

$$
\begin{aligned}
& p=p_{\exp }(t) \\
& \frac{\partial h}{\partial t}=\frac{1}{\rho} \frac{\partial p}{\partial t} \\
& p=\frac{\rho}{M_{\text {mean }}} R T \\
& M_{\text {mean }}=\left(\sum_{i=1}^{n_{s}} Y_{i} / M_{i}\right)^{-1} \\
& h=\sum_{i=1}^{n_{s}} Y_{i} h_{i}(T) \\
& h_{i}(T)=\frac{H_{i}(T)}{M_{i}} \\
& \frac{\partial Y_{i}}{\partial t}=\frac{M_{i}}{\rho} \omega_{i}
\end{aligned}
$$

where $p$ is the pressure, $h$ the specific enthalpy (in $\mathrm{J} / \mathrm{kg}$ ) of the gas mixture, $\rho$ the mass density, and $M_{\text {mean }}$ the mean molar mass of the gas mixture. $R=8.3145 \mathrm{~J} /(\mathrm{mol} \cdot \mathrm{K})$ is the universal gas constant, the $Y_{i}$ $\left(i=1, \ldots, n_{\mathrm{s}}\right.$, where $n_{\mathrm{s}}$ is the number of chemical species) are the mass fractions of species that participate in the chemical reactions, the $h_{i}$ and $M_{i}$ are the specific enthalpies and molar masses of species $i$, respectively. The temperature dependent specific (mass-based) enthalpies $h_{i}$ of the species are computed from the molar enthalpies $H_{i}(T)$, which are in turn computed from NASA polynomials. The $\omega_{i}$ are the rates of formation of species due to chemical reactions (in $\mathrm{mole} /\left(\mathrm{m}^{3} \cdot \mathrm{s}\right)$ ). To describe the chemical rates of formation or consumption as a function of species concentrations, pressure and temperature, a chemical mechanism tuned to describe the low-temperature kinetics of iso-octane/n-heptane was employed $[13,14]$. This model can, in short, be specified as an adiabatic, constant-mass, single-zone model with detailed chemistry and a prescribed temporal development of the cylinder pressure. To set up the equation system (1.) for given initial and boundary conditions and specified thermodynamical and chemical kinetics data, and to compute the numerical solution of the equations, the computer code HOMREA (see [14], and references therein) was used.

The simulation starts at bottom dead center, with initial values of temperature $T(0), p(0)$ and $Y_{\mathrm{i}}(0)$. This specification of the initial state also defines the specific enthalpy, density and mean molar mass of the mixture. A set of simulations was performed, with initial conditions varying in a range chosen to represent a simple test en- gine (see below). The simulations yield the temporal development of species concentrations and of temperature during the compression and expansion stroke.

In Figure 2, sample results of the simulations are shown. As outlined in the "Methodology" section, this plot corresponds to the left branch of the curve in Figure 1, with formaldehyde as intermediate species. The points in the diagram represent a set of 50 different calculations, each one performed using a different measured pressure trace as a temporal boundary condition. Also, different initial temperatures were used in the calculations, ranging from $290 \mathrm{~K}$ to $350 \mathrm{~K}$. Each data point represents an individual time step in a simulation, and there are approximately 100 points per one simulation visible. Despite the different initial and temporal boundary conditions, all calculated points are close to the same curve, which represents the formation of formaldehyde (compare the qualitative sketch in Figure 1) in the pre-ignition phase. To the right, towards higher temperatures, the starting destruction of formaldehyde by reactions that lead to combustion is seen by the curvature of the $\mathrm{CH}_{2} \mathrm{O}$ vs. $T$ curve, indicating that a maximum of formaldehyde concentration is close. Compare this to the qualitative sketch shown in Figure 1.

In the semi-logarithmic diagram of Figure 2, the data set appears to represent a linear correlation during the preignition phase, so that the underlying relation may be approximately described by the formula

$$
\left[\mathrm{CH}_{2} \mathrm{O}\right]=A \exp B T \text {, }
$$

where the brackets [...] here denote concentrations, in mole $/ \mathrm{m}^{3}$. The quantities $A$ and $B$ can be determined from the correlation. In logarithmic form, this reads

$$
\log \left[\mathrm{CH}_{2} \mathrm{O}\right]=\log A+B T \text {. }
$$

$B$ is the slope of the temperature-log $\left[\mathrm{CH}_{2} \mathrm{O}\right]$ correlation curve. As shown in Figure 2, $A$ and $B$ depend only weakly on the initial temperature $\left(T_{0}\right)$ and the temporal boundary condition (pressure trace) chosen for the simulation.

Let us consider two spatial sites (termed 1 and 2) in the combustion chamber which feature slightly different temperatures $T_{1}$ and $T_{2}$ at a given instant of time. This represents a temperature fluctuation in the combustion chamber. Due to this temperature difference $\Delta T=T_{2}-T_{1}$, the sites will display a difference in formaldehyde concentration. If we consider the difference of the logarithms of these concentrations, $\Delta \log \left[\mathrm{CH}_{2} \mathrm{O}\right]=\log \left[\mathrm{CH}_{2} \mathrm{O}\right]_{2}$ $\log \left[\mathrm{CH}_{2} \mathrm{O}\right]_{1}$ we find from eq. (3.).

$$
\frac{\Delta \log \left[\mathrm{CH}_{2} \mathrm{O}\right]}{\Delta T}=B
$$

The quantity $A$ disappears after forming the differences; it is therefore immaterial for our purpose of determining temperature fluctuations. For measurements of absolute 
temperature (rather than local temperature fluctuations), $A$ would have to be known. This would require a measurement of the absolute formaldehyde concentration, which is not easy to perform (albeit in principle possible) in an engine, especially not with the required high accuracy.

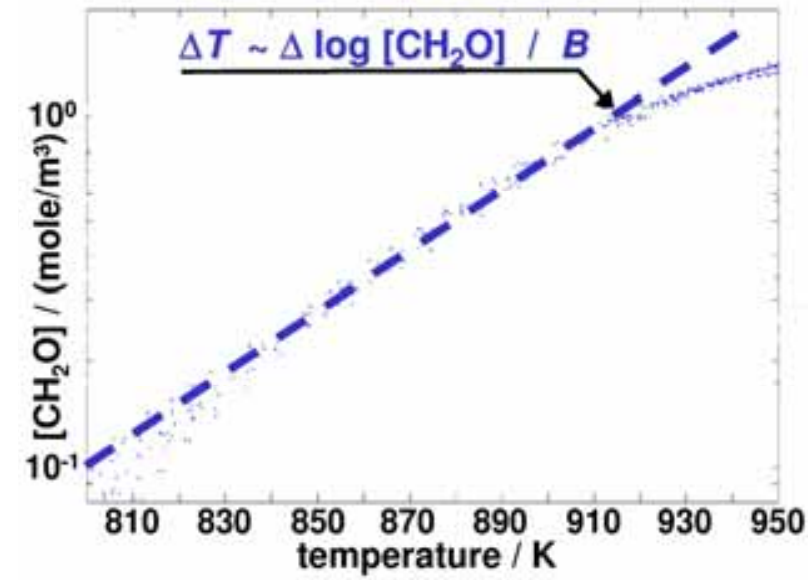

Figure 2 Computed correlation between formaldehyde concentration (logarithmic scale) and temperature during the compression stroke. The dots represent data obtained from simulations of several engine cycles with different initial and boundary conditions.

Rewriting eq. (4.) yields a relationship that allows estimating temperature fluctuations from fluctuations of the formaldehyde concentration:

$$
\Delta T=\frac{\Delta \log \left[\mathrm{CH}_{2} \mathrm{O}\right]}{B}
$$

For a given fluctuation of the formaldehyde concentration, a smaller value of $B$ will result in a larger fluctuation of the temperature.

\section{LIF-SIGNAL - CONCENTRATION CORRELATIONS}

Given a fixed formaldehyde concentration, laserintensity and detection efficiency of the employed experimental setup, the measured LIF-intensity $I$ depends on temperature, pressure and mixture composition. To infer spatial temperature fluctuations from LIF-signal fluctuations, we need to consider the spatial variation of the LIF-signal due to local variations of temperature and species concentrations. When considering various effects that influence the LIF-signal, it is important to note that in our case we are interested only in spatial variations of these effects, and not on their absolute magnitude. This fact significantly alleviates many of the problems that are often encountered in attempts to measure absolute quantities (like temperature or species concentrations) by laser-induced fluorescence in engines.

The local chemical composition of the gas bath in which the fluorescence emitter formaldehyde is embedded has mainly two effects on the LIF-signal. First, collisional broadening of absorption lines alters the effective absorption cross section of formaldehyde, and therefore indirectly also the LIF emission strength. Furthermore, collisional quenching of laser-excited formaldehyde molecules by other molecules (as well as similar effects that compete with the spontaneous emission of photons) will affect the LIF-signal.

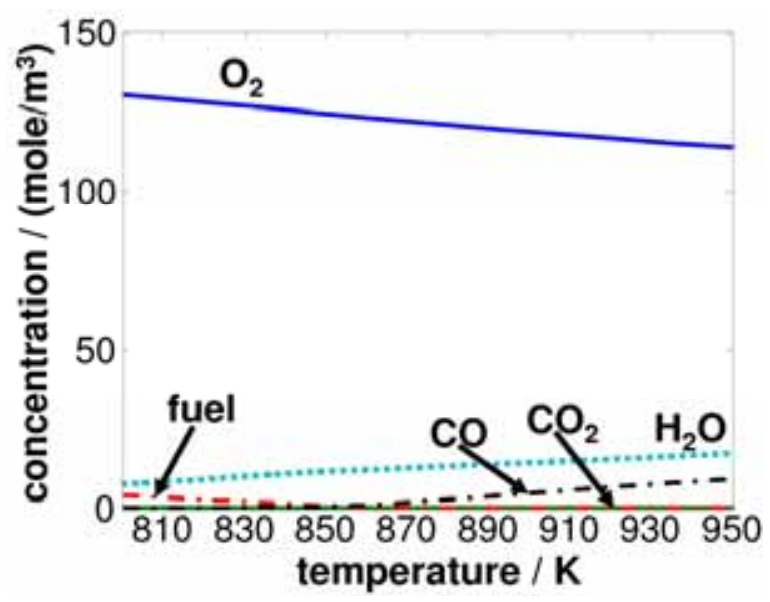

Figure 3 Computed concentrations of various major species vs. temperature, during the compression stroke. This corresponds to Figure 2, but only one out of the 50 calculations (for $T_{0}=300 \mathrm{~K}$ ) is shown here. Note that $\left[\mathrm{CO}_{2}\right]$ is too small to be distinguished from zero in this graph.

The strength of the collisional quenching can be expressed by the quenching rate $Q$. $Q$ is a concentrationweighted sum over all species of the form [16]

$$
Q=\sum_{i=1}^{n_{s}} c_{i} \sigma_{i} v_{i}
$$

where the $c_{i}$ are the concentrations of the species (index $i$ runs over all species), the $\sigma_{i}$ the quenching cross sections and the $v_{i}$ the average relative velocities of the colliding molecules. Equation (6.) contains several implicit temperature dependencies: The $v_{i}$ contain a term that is proportional to $T^{1 / 2}$, and the $\sigma_{i}$ are in general also temperature-dependent. To our knowledge, for most collider-species, no explicit information about quenching cross sections is available for formaldehyde quenching. One can estimate, however, that $Q$ will not vary considerably if no large spatial variations of temperature and major species concentrations occur in the combustion chamber. For this estimation, Figure 3 displays the calculated concentrations of the major species $\mathrm{O}_{2}$, fuel, $\mathrm{CO}$, $\mathrm{CO}_{2}$, and $\mathrm{H}_{2} \mathrm{O}$ in the unburned gas, again, during the phase prior to auto-ignition. The data are taken from the same simulations that were also used for creating Figure 2 ; however, only the result from one out of the 50 calculations is shown here for clearer presentation. Not shown in the graph is nitrogen $\left(\mathrm{N}_{2}\right)$, which displays virtually no change of concentration in the considered range. $\mathrm{CO}_{2}$ is present only in negligible amounts here, since we 
consider a phase before the onset of combustion. The graph for $\mathrm{CO}_{2}$ is therefore hardly distinguishable from zero. It is seen that the variation of major species concentrations in the considered temperature range is small. The effect of collisional quenching on the LIFsignal will also display only small variation with temperature. To obtain a more quantitative assessment of the variation of the effect of quenching, let us consider quenching of nitric monoxide (NO) as a representative example. For this molecule, data for computing quenching cross sections (and therefore, for numerically evaluating eq. (6.)) are available $[17,18]$. Using these spectroscopic data and the computed variation of major species concentrations and temperature (shown in Figure 3 ), one obtains a relative variation of $Q$ within the considered range of below $25 \%$. Although the quenching behaviour of formaldehyde is certainly not the same as that of $\mathrm{NO}$, the variation of formaldehyde quenching with temperature will not be entirely different from that of NO, but will be within the same order of magnitude. We conclude that it is also a small variation; the term "small variation" here is to be understood as "small variation compared to the variation of formaldehyde concentration due to chemistry".

A similar argument also applies to the variation of pressure broadening: The contribution to this effect is mainly from major species, and therefore the variation of the effect with temperature is not significant compared to the strong variation of formaldehyde concentration with temperature due to chemical reactions. Doppler broadening of spectral lines [16] scales with the square root of temperature (a comparatively weak dependence), and is of minor influence in the high-pressure environment of an engine, where the width of spectral lines is dominated by pressure broadening. This is especially true if, as in our case, only the unburned gas is considered.

There remains to consider the effect of temperature onto the thermal population of the laser-excited ground states of formaldehyde. To estimate the relative importance of these effects for the LIF-signal fluctuations, spectroscopic calculations were performed. For this, the thermal population of the laser-excited ground states of formaldehyde was computed using vibrational frequencies of the formaldehyde molecule [19] and by using the program WANG [20] for computing the rotational energy levels.

Due to the high pressures in engines, pressure broadening of absorption lines is significant. For formaldehyde, the spectral separation of rotational lines is very narrow, and absorption lines strongly therefore strongly overlap. Therefore, thermal populations of several ground states involved in transitions near $339.3 \mathrm{~nm}$ were studied in the model. The result of this analysis is shown in Figure 4, where the variation of thermal ground state population, the variation of quenching (represented here by a $T^{0.5}$ law), as well as the product of these variations is shown.

All quantities are normalized so that they are equal to 1 at $900 \mathrm{~K}$. Since for our purpose it suffices to consider the variation (not the absolute values), this normalisation is immaterial. For comparison, the variation of formaldehyde concentration with temperature, taken from the engine simulations with detailed chemistry (see the section about the temperature/concentration correlations) is shown. It is seen that the thermal population of the laserexcited ground states varies much less than the concentration of formaldehyde in the range from $700 \mathrm{~K}$ to $1100 \mathrm{~K}$. Also, the variation of the collisional quenching effect with temperature is considerably smaller than the variation of formaldehyde number density. The combined effect of variation of quenching and the variation of thermal ground state population onto the LIF-signal is by one order of magnitude less than the variation of formaldehyde number density due to chemistry.

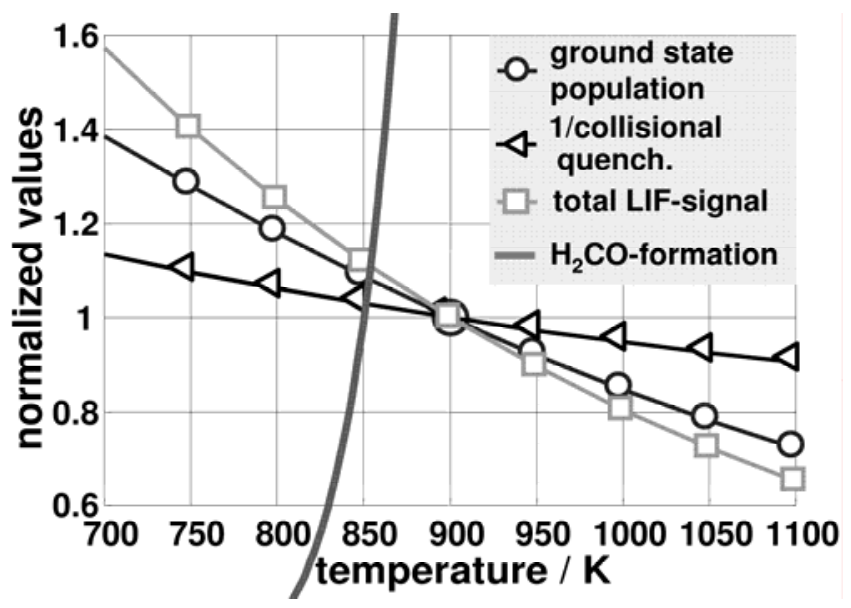

Figure 4 Computed effect of temperature variations on various quantities that affect the strength of Laser-Induced Fluorescence signals. The total LIF signal dependence shown here (square symbols) is for a fixed number of $\mathrm{CH}_{2} \mathrm{O}$ molecules.

The combined effect of these temperature dependencies onto the fluorescence intensity I (LIF-signal) for a fixed formaldehyde concentration can be approximated well by an exponential function of the form $D \exp (E T)$, where $D$ and $E$ are temperature-independent quantities. This approach represents the LIF-signal dependence shown in Figure 4 (curve marked with squares). Note that this term is to be understood as the LIF-signal for a given (fixed) amount of formaldehyde molecules; it does not include the variation of formaldehyde number density due to chemical formation in temperature dependent chemical reactions.

The resulting overall dependence of the measured fluorescence signal includes the variation of formaldehyde concentration with temperature due to chemical reactions and the dependence due to spectroscopy. It can be expressed by the product

$$
I=A \exp (B T) D \exp (E T) K
$$

where $K$ is a constant that describes the dependence of the signal on details of the experimental setup, like the intensity of the laser used to excite the fluorescence, the 
collection efficiency of the employed optical line, the detector efficiency, and so on. Note that $K$ does not contain any dependence of spatially varying spectroscopical properties; these are expressed by the $D \exp (E T)$ term. $K$ can display a spatial variation, for example due to absorption of the exciting laser beam. Absorption was measured in previous investigations [14] with the same engine and experimental conditions; in our case, it is too weak to be significant for the evaluation of temperature fluctuations.

After forming the logarithm, equation (7.) becomes

$$
\log I=\log A+B T+\log D+E T+\log K
$$

According to our approach, $A, B, D, E$ and $K$ do not depend on temperature. Therefore, the difference of the logarithm of the LIF-signals from two different spatial sites 1 and 2 may be expressed as

$$
\begin{gathered}
\Delta \log I=\log I_{2}-\log I_{1}=B\left(T_{2}-T_{1}\right)+E\left(T_{2}-T_{1}\right) \\
=B \Delta T+E \Delta T,
\end{gathered}
$$

since $\log A, \log D$ and $\log K$ cancel out by forming the differences. We then get

$$
\Delta T=\Delta \log I /(B+E)
$$

This equation allows deriving temperature fluctuations $\Delta T$ directly from fluctuations of the measured LIF-signal. To evaluate eq. (10.), knowledge of the quantities $B$ and $E$ is required. These can in our case be determined reasonably well by simulations of chemical kinetics and spectroscopic modelling, as outlined above.

The accuracy of the method is, on the one hand, determined by the accuracy of $B$ and $E$, which in turn depends on the accuracy of available data on which chemical kinetics simulations $(B)$ and spectroscopic calculations $(E)$ are based.

Furthermore, fluctuations of the signal / may also be due to signal noise and not genuinely due to fluctuations of the formaldehyde concentration. If signal noise contributes to the quantity $\Delta \log I$, there will result too high values $\Delta T$ from eq. (10.). However, the influence of signal noise can be determined (and then also be corrected for) by using the employed laser and detection setup for measuring the LIF-signal field from formaldehyde with a spatially homogeneous concentration field, e.g. in a test cell, as shown below.

\section{EXPERIMENTAL}

Laser induced fluorescence experiments of native formaldehyde in the unburned gas were performed in an optically accessible test engine. The studies aimed at checking the feasibility of the method for temperature fluctuation diagnostics and at obtaining reasonable estimates for temperature fluctuation amplitudes in a nominally homogeneous engine.
Details of the engine are explained in [14] and references therein, so only a short account and Table 1 will be given here. The engine (ILO L372, two-stroke Otto with external mixture formation) was operated with a mixture of iso-octane and $n$-heptane (95\%/5\% vol./vol.). Some more information about the engine is comprised in Table 1. The crank shaft of the engine was coupled to a strong dynamometer during operation, which served to drive and brake the engine to maintain a constant engine speed of $1000 \mathrm{rpm}$. Only every $5^{\text {th }}$ cycle was ignited, so that the remaining four cycles served for scavenging residual exhaust gas from the cylinder.

\begin{tabular}{|r|l|}
\hline type & $\begin{array}{l}\text { ILO L372 two-stroke } \\
\text { Otto-Engine }\end{array}$ \\
\hline fuel & $\begin{array}{l}\text { iso-octane/n-heptane } \\
\text { mixture }(95 / 5 \text { vol./vol.) }\end{array}$ \\
\hline equivalence ratio & $\Phi=1$ \\
\hline mixture formation & carburettor \\
\hline bore $\times$ stroke & $80 \mathrm{~mm} \times 74 \mathrm{~mm}$ \\
\hline $\begin{array}{r}\text { geometrical compression } \\
\text { ratio }\end{array}$ & $8.5: 1$ \\
\hline
\end{tabular}

Table 1 Engine data

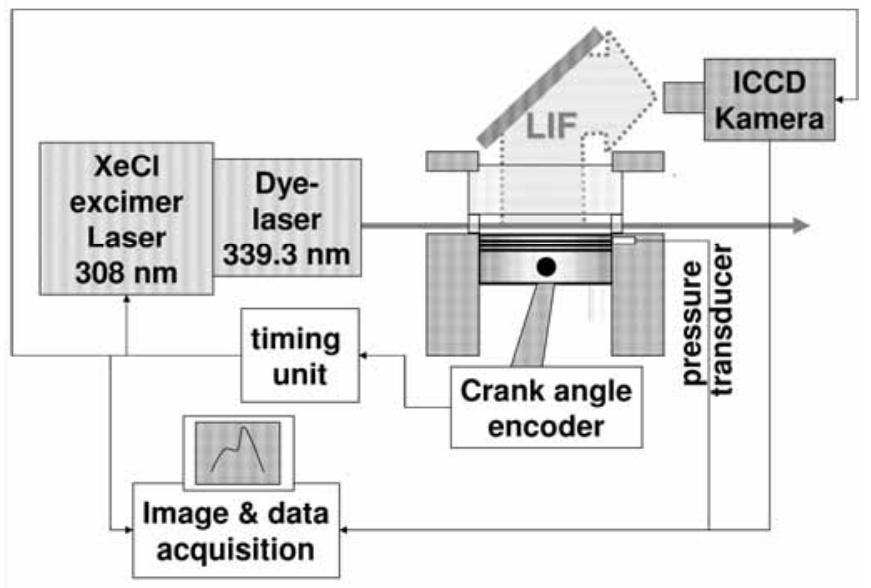

Figure 5 Sketch of the experimental setup for detecting LIF of native formaldehyde in the unburned gas of the optically accessible IC engine

The engine was equipped with a crank angle encoder that allowed synchronizing other devices (laser, camera, data acquisition) with the engine cycle. The engine has a pan-shaped combustion chamber with a diameter of $80 \mathrm{~mm}$ and a clearance height of $4 \mathrm{~mm}$ at TDC. The combustion chamber provides optical access by a quartz glass ring with a height of $4 \mathrm{~mm}$ and by a quartz glass block. The fuel/air mixture in the engine has been checked to be very homogeneous with respect to the fuel/air ratio $[13,14,15]$. The checks were performed by doping the fuel of the engine with acetone, and by then visualizing planar acetone-LIF maps excited by $\mathrm{XeCl}$ Excimer radiation at $308 \mathrm{~nm}$. 
Detection of native formaldehyde in the unburned gas of the engine was performed using the setup shown in Figure 5 . The same setup has been described in [13, 14]; we will give a short account here only. A XeCl excimer laser (Lambda Physik LPX 200, emission at 308 $\mathrm{nm}$, pulse duration $20 \mathrm{~ns}$ ) was used to pump a dye laser (Lambda Physik FL 3002). The output wavelength of the dye laser was tuned near to $339 \mathrm{~nm}$, so that it spectrally coincided with the center of the $2{ }_{0}{ }^{1} 4_{0}{ }^{1}$ vibrational absorption feature of formaldehyde in the electronic $A^{1} A_{2}-X^{1} A_{1}$ transition [21, 19, 22]. The output of the laser was shaped into a cylindrical beam with a diameter of approx. $300 \mu \mathrm{m}$ by means of lenses and a pinhole. The beam was shone into the combustion chamber of the engine, in a direction parallel to the piston surface. The beam crossed the unburned gas of the engine and caused native formaldehyde in the endgas to fluoresce in the laser-illuminated region. The fluorescence was detected through a thick quartz glass window which served as the cylinder head. During experiment preparation, it was made sure that formaldehyde was the only substance that contributed to the LIF-signal by recording a fluorescence emission spectrum [14] in the running engine.

In the actual experiments, an ICCD camera (Proxitronic NANOCAM, 8 bit dynamical range, $512 \times 512$ pixels) was used to detect the formaldehyde LIF trace. The camera was equipped with a UV lens (UV Nikkor, focal length $200 \mathrm{~mm}$ ), and a colour glass filter (Schott GG 385) to block laser scatter. Experiments were performed at several crank angles, with 36 single-shot LIF-images recorded in consecutive fired engine cycles.

\section{RESULTS}

\section{LIF INTENSITY FLUCTUATIONS}

Figure 6 shows on top the intensity (greyscale) image of a formaldehyde LIF trace, together with a grey value bar. It was recorded in the unburned gas of the operating engine at TDC, at an engine speed of $1000 \mathrm{rpm}$. The trace was recorded near the middle of the combustion chamber. Note that at TDC the height of the pan-shaped combustion chamber is $4 \mathrm{~mm}$ only, so that the laserbeam used to excite the fluorescence trace is no further than $2 \mathrm{~mm}$ away from the piston crown and the quartz glass block serving as the cylinder head (cf. Figure 5, which displays the position and orientation of the laser beam within the combustion chamber).

In the diagram below, the spatial profile of the normalized LIF intensity fluctuation is depicted. The profile is taken along the direction of the laser beam. Symbol $x$ denotes the spatial location along the profile. The normalized LIF intensity fluctuation is defined as $\Delta I(x) / I_{\mathrm{m}}$, where $I_{\mathrm{m}}$ is the average LIF intensity along the profile and $\Delta l(x)=I(x)-I_{m}$. The profile $I(x)$ was obtained by column-wise summation of the image grey values, i.e., by binning all pixel grey values in vertical direction. This binning procedure reduces the influence of noise onto the resulting LIF-profile; furthermore, it removes the influence of laser-beam profile inhomogeneities. It is seen that considerable fluctuations of the LIF-intensity profile exist, even though the fuel/air ratio in this engine is very homogeneous. The intensity fluctuations display pronounced large scale structures, with amplitudes clearly exceeding the noise level in our measurement. The "wavy" appearance of the horizontal border of the LIFtrace is mainly a consequence of the intensity fluctuations (due to the reproduction of the image as a grey value map, darker regions appear thinner).

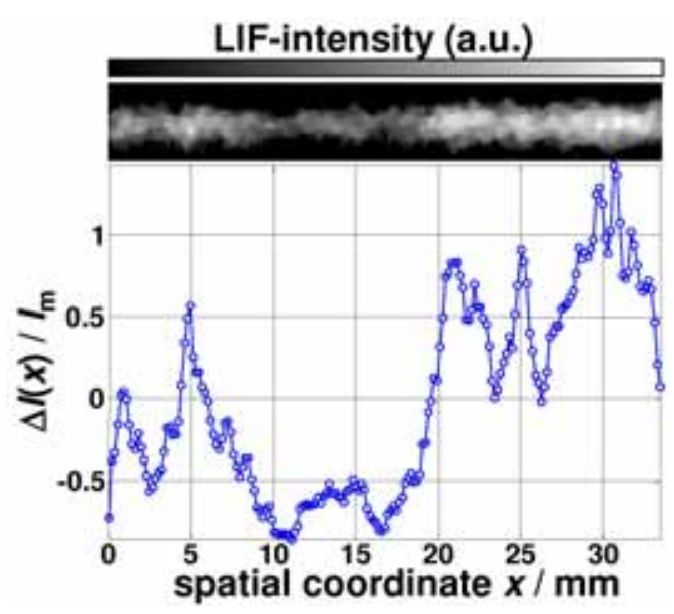

Figure 6 Measured LIF-intensity profile of formaldehyde, recorded in the unburned gas of the test engine at TDC. Top: Image of a formaldehyde fluorescence trace, with corresponding grey colour bar placed above. Bottom: The resulting normalized grey value fluctuation profile. $I_{\mathrm{m}}$ is the mean value of the signal.

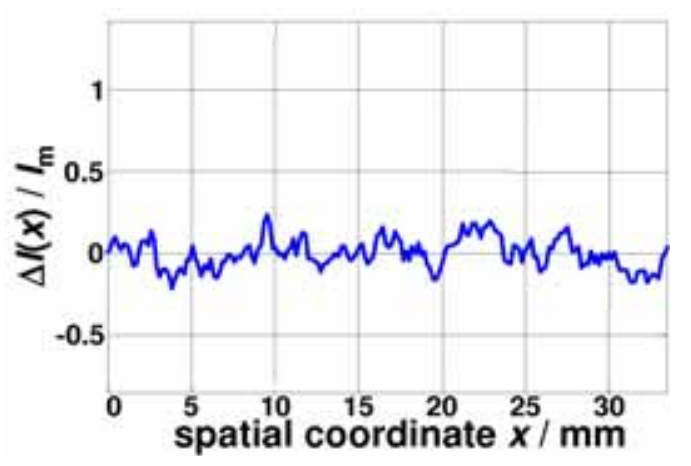

Figure 7 Normalized intensity fluctuation profile of the LIF-signal recorded in a test cell filled with a homogeneous $\mathrm{CH}_{2} \mathrm{O}$ lair mixture. For comparison, axes scaling is the same as in Figure 6.

To provide a notion on the spatial LIF intensity variation that is due to noise, Figure 7 shows for comparison a LIF-profile that was measured in a static test cell filled with a formaldehyde/air mixture. This profile was measured with the same setup that was also used for the engine measurements, at comparable signal strengths. Since the formaldehyde in the test cell is homogene- 
ously distributed, the fluctuation in the LIF-signal here is entirely due to signal noise. It is seen that the fluctuation amplitude is much smaller than in the case of the engine LIF-measurements. Also, in the LIF-profiles measured in the test cell, there do not appear any structures of larger scale (in the range of $1 \mathrm{~cm}$ ) like the ones that are visible in the engine measurements. Formaldehyde LIF intensity fluctuations in the unburned gas of IC engines have been observed also by other groups in similar experiments [23]; they can also be observed with laser induced fluorescence of intermediate species other than formaldehyde [13]. The fluctuations are essentially due to local temperature variations which cause local variations in the rate of formaldehyde formation via the Arrhenius-like temperature dependence of chemical reaction velocities. It is seen that the fluctuations can have considerable geometrical size, ranging from a few $\mathrm{mm}$ to the cm-scale.

\section{ESTIMATION OF TEMPERATURE FLUCTUATION AMPLITUDES}

We can use equation (10.) to infer temperature fluctuation amplitudes from the measured LIF intensity variation. The required values for the quantities $B$ and $E$ can be determined according to the procedure outlined above. For the conditions valid in our engine experiments, and using the iso-octane/n-heptane mechanism we employed for our engine-simulations, one obtains values of $B=2 \times 10^{-2} \mathrm{~K}^{-1}$ and $E=2 \times 10^{-3} \mathrm{~K}^{-1}$. In the results presented in this paper, we will use these values for the evaluation, unless otherwise noted. In Figure 8, the transformation from the normalized LIF-intensity profile (top diagram) to a temperature fluctuation profile (bottom) is shown. Due to the nonlinear relationship between temperature and LIF-intensity (according to equation (10.)), the shapes of the two curves are not identical. They are, however, similar in that the spatial structure of LIF-fluctuations and temperature fluctuations are the same. Exactly like the LIF-fluctuations, the geometrical size of the temperature fluctuations ranges from the $\mathrm{mm}$ to the $\mathrm{cm}$ scale.

According to the laws of error propagation, the uncertainty of the resulting values for the temperature fluctuations $\Delta T$ is due to uncertainties in the term $B+E$ and in $\Delta \log I$. The value for $B$ results from a linear fit to the correlation curve shown in Figure 2; it will vary depending on where exactly the linear fit is applied, though this variation is quite small in a wide temperature range. Also, the values for $B$ vary when different chemical mechanisms are used for the computation of the $\left[\mathrm{CH}_{2} \mathrm{O}\right]-$ $T$ correlation curve. A simple check with different iso-octane/n-heptane mechanisms yielded values of $B$ that differed by a factor of about 2 from the values obtained with our mechanism. For all mechanisms we studied, $B$ is large compared to the value for $E$, so that uncertainties in $E$ are of minor importance in our case. Uncertainties in $\Delta \log I$ arise mostly from the contribution of noise to the LIF-signal fluctuations. In our case, this contribution is quite small, though not completely negligible (compare Figure 6 and Figure 7).
Signal noise limits the minimal temperature fluctuation that still can be detected with the technique. We can estimate this detection limit by converting the observed LIF-fluctuation due to noise (measured in a test cell with homogeneous formaldehyde distribution, Figure 7) into a temperature fluctuation, according to eq. (10.). This yields a fluctuation amplitude of about $2 \mathrm{~K}$, using the value of $B$ determined with our mechanism. Taking into account the uncertainties in $B$ described above yields a value of about $4 \mathrm{~K}$ for the detection limit (using the "worst" observed value for $B$ ). Lower temperature fluctuations would cause LIF fluctuations that are too small to be distinguishable from the measurement noise. Above this detection limit, the accuracy in estimating the temperature fluctuation amplitudes is in our case mainly limited by the accuracy of the value for $B$. According to the considerations made above, an uncertainty of a factor of about two for the temperature amplitudes has then to be taken into account. Uncertainty that comes from signal noise (in our case, around $2 \mathrm{~K}$ to $4 \mathrm{~K}$ ), will occur additionally.

Even with the limits of accuracy and detectability, it is obvious from the measurements that considerable temperature fluctuations exceeding $10 \mathrm{~K}$ exist in an engine with a nominally homogeneous load. The fluctuation amplitudes are, on the other hand, by more than one order of magnitude smaller than the corresponding mean value of the gas temperature towards the end of the compression phase (prior to auto-ignition); in this sense, the fluctuations are "small".

From the information obtained by repeatedly acquiring LIF-traces in the running engine, a statistical distribution of the temperature fluctuation amplitudes can be determined. A probability density function (PDF, graphically represented here as a normalized histogram) of fluctuations recorded in a total 500 laser-shots, all under nominally identical conditions, is shown in Figure 9. The PDF was computed in the following way: First, from 500 single-shot LIF traces, the corresponding 500 LIFfluctuation profiles were computed by the column-wise binning procedure described above. Each profile contains the fluctuations at the individual bins in spatial direction. All these fluctuation values (from all bins of all 500 single-shot experiments) were used as the statistical ensemble from which the PDF was determined. Of course, by definition, the mean value of the fluctuations in this ensemble is zero. It is seen from the PDF that the temperature fluctuation distribution is symmetric. Fitting a Gaussian normal distribution to the PDF yields a full width at half maximum (FWHM) of $14 \mathrm{~K}$.

The existence and geometrical size of the fluctuations, as derived from the formaldehyde LIF signals, is in accordance with earlier measurements that were performed in the same engine, using detection of (not uniquely identified) intermediate species by LIF. Excitation in these experiments was performed with a XeCl-excimer laser [13]. 


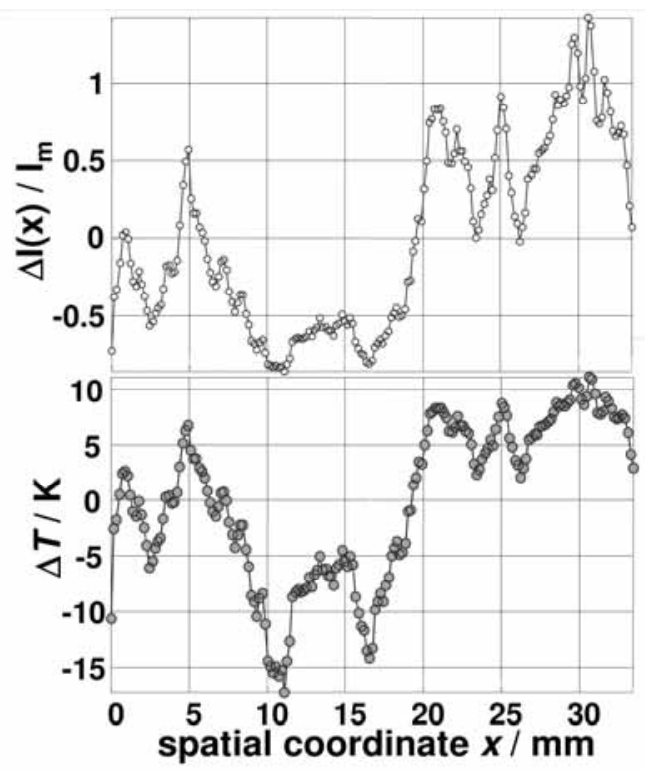

Figure 8 Normalized LIF intensity fluctuation profile (top, same as in Figure 6) and the corresponding derived temperature fluctuation profile (bottom).

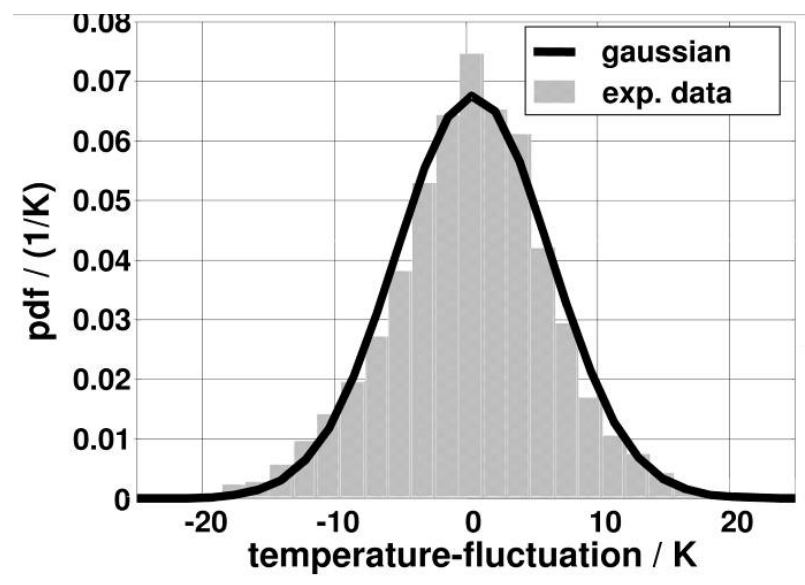

Figure 9 Grey bars: Probability density function (PDF) of fluctuation amplitudes, sampled from 500 single-shots in different engine cycles. A least-squares fit (Gaussian) to the PDF is also shown (solid line).

However, for the unidentified intermediate species, the interpretation of these LIF signals in terms of temperature fluctuation amplitudes was problematic due to the lack of knowledge about spectroscopic behaviour of most intermediates and the unclear identity of the fluorescence emitter. For the measurements based on formaldehyde, this situation is considerably better.

\section{SUMMARY AND CONCLUSIONS}

Laser-induced fluorescence of native formaldehyde was used to detect and semi-quantitatively determine spatial temperature fluctuations in the unburned gas of a simple test IC engine. The detection method uses native for- maldehyde in the unburned gas as an extremely sensitive tag for temperature fluctuations. The high sensitivity is due to the strong, Arrhenius-like temperature dependence of the chemical reactions that lead to the formation of formaldehyde, typically occurring in the late compression phase of the engine cycle. In this phase, the strongly temperature dependent rate of chemical formation induces a strong correlation between formaldehyde concentration and temperature. After transforming the formaldehyde concentration fluctuations into LIF-signal fluctuations by spectroscopic calculations, this correlation can be used to transform the observed spatial formaldehyde LIF-signal fluctuations into temperature fluctuations.

The method is applicable in environments where no strong local inhomogeneities of the equivalence ratio exist. To infer temperature fluctuation amplitudes from the observed formaldehyde LIF-fluctuations, information about the variation of formaldehyde number density with temperature (due to chemical reactions) is required. We obtained this information from numerical simulations of a simple model representing the cylinder-load during the compression and pre-ignition phase. To apply the method in an actual experiment, correlations between formaldehyde LIF-signals and temperature are needed. These are obtained by computing the variation of spectroscopic properties of formaldehyde with temperature. Important effects that affect the LIF-signal, like the temperature-dependent thermal population of the laserexcited ground state, and the temperature- and composition dependent quenching of fluorescence signals, are estimated based on spectroscopic computations.

The results show that fluctuations with amplitudes well above $10 \mathrm{~K}$ can exist even in a nominally homogeneous engine. The geometrical size of these fluctuations can also be considerable. Values of up to $1 \mathrm{~cm}$ were observed in a cylinder with a diameter of $8 \mathrm{~cm}$. As a limit for the minimum temperature fluctuation amplitude that can be detected with the method, (limitation due to noise in the LIF-signal), $2 \mathrm{~K}$...4K can be estimated, based on (and valid for) the signal to noise ratio in our experiment. In our engine, the fluctuation amplitude has a symmetric, Gaussian statistical distribution. The width (FWHM) of this distribution can be estimated, based on the correlation between formaldehyde LIF-signal and temperature we obtained by our computations, to be $14 \mathrm{~K}$. Again, due to the uncertainties associated with the determination of this correlation, this value should be regarded as semiquantitative, within a factor of about two.

It has to be stressed that the method we describe here is not capable of measuring absolute temperatures in the unburned gas with an accuracy as good as $10 \mathrm{~K}$ and better - the method can detect spatial temperature fluctuations with high sensitivity, and provide a semiquantitative estimate of their amplitude. No detailed information about the mean value of the temperature can be inferred, however. To obtain the complete temperature field (both the mean and fluctuations), complementary information from other sources is required. 


\section{REFERENCES}

1. A. K. Oppenheim. The knock syndrome - Its cures and its victims. SAE Transactions, volume 93, paper no. 841339, section 5, Society of Automotive Engineers, 1984, 874-883

2. J. Warnatz, U. Maas, R.W. Dibble, Combustion, Springer, 2001

3. R. H. Thring, Homogeneous Charge Compression Ignition $(\mathrm{HCCl})$ engines, SAE Paper No. 892068, 1989

4. R. H. Stanglmaier, C. E. Roberts, Homogeneous charge Compression Ignition $(\mathrm{HCCl})$ : Benefits, Compromises and Future Engine Applications, SAE 1999-01-3682

5. S. Hajireza, F. Mauss, B. Sunden, Effect of Inhomogeneities in the End Gas Temperature Field on the Autoignition in SI Engines, SAE Paper 2000-010954 (2000)

6. J. Bood, P. E. Bengtsson, F. Mauss, K. Burgdorf, I.G. Denbratt, Knock in Spark-Ignition Engines: EndGas Temperature Measurements Using Rotational Cars and Detailed Kinetic Calculations of the Autoignition Process, SAE Paper No. 971669 (1997)

7. M. Sjöberg, J. E. Dec., A. Babajimopoulos., D. Assanis, Comparing Enhanced Natural thermal stratification against retarded combustion phasing for smoothing $\mathrm{HCCl}$ heat-release rates, SAE paper No. 2004-01-2994, 2004

8. J. E. Dec, W. Hwang, M. Sjöberg, An Investigation of Thermal Stratification in $\mathrm{HCCl}$ engines using Chemiluminescence Imaging, SAE Paper No. 200601-1518, 2006

9. S. Einecke, Ch. Schulz, V. Sick, R. Schießl, A. Dreizler, U. Maas, Two-Dimensional Temperature Measurements in An SI Engine Using Two-Line Tracer LIF, SAE Paper No. 982468 (1998)

10. M. Richter, J. Engström, A. Franke, M. Alden, A. Hultqvist, B. Johansson, The Influence of charge Inhomogeneity on the $\mathrm{HCCl}$ combustion process, SAE Paper 2000-01-2868, 2000

11. B. Bäuerle, F. Hoffmann, F. Behrendt, J. Warnatz, Detection of hot spots in the end gas of an internal combustion engine using two-dimensional LIF of formaldehyde, Proc. Comb. Inst. 25, p. 135-141 (1994)

12. G. Sarner, M. Richter, M. Alden, L. Hlidingsson, A. Hultqvist, B. Johansson, Simultaneous PLIF Measurements for Visualization of Formaldehyde- and Fuel- Distributions in a DI HCCI Engine, SAE Paper 2005-01-3869

13. R. SchießI, U. Maas, Analysis of Endgas Temperature Inhomogeneities in an SI Engine by Laser-
Induced Fluorescence. Comb. Flame, 133: 19-27, 2003.

14. R. Schießl, P. Pixner, A. Dreizler, U. Maas: Formaldehyde formation in the endgas of Otto engines: Numerical simulations and quantitative concentration measurements. Combustion Science and Technology, 149:339-360, 1999.

15. R. Schießl, A. Dreizler, U. Maas, A. J. Grant, P. Ewart: Double-Pulse PLIF imaging of Self-Ignition Centers in an SI engine. SAE paper 2001-01-1925, 2001

16. A. C. Eckbreth, Laser Diagnostics for Combustion Temperature and Species, $2^{\text {nd }}$ ed.,Gordon and Breach, ISBN 90-5699-532-4, p. 400 (1996)

17. P. H. Paul, J. A.. Gray, J. L. Durant, J. W. Thoman, A Model for Temperature-Dependent Collisional Quenching of NO $A^{2} \Sigma^{+}$, Appl. Phys. B 57, pp. 249259 (1993)

18. P. H. Paul, C. D. Carter, J. A. Gray, J. L. Durant, J. W. Thoman, M. R. Furlanetto, Correlations for the NO $A^{2} \Sigma^{+}\left(v^{\prime}=0\right)$ Electronic Quenching Cross-section, Sandia Report SAND94-8237 UC-1423, Sandia National Laboratory, Livermore, CA (1995).

19. D. J. Clouthier and D. A. Ramsay, The spectroscopy of formaldehyde and thioformaldehyde, Ann. Rev. Phys. Chem., 43: 31—58, 1983

20. D. Luckhaus, M. Quack, The Far Infrared Pure Rotational Spectrum and the Coriolis Coupling between $\mathrm{v}_{3}$ and $\mathrm{v}_{8}$ in $\mathrm{CH}^{35} \mathrm{ClF} 2$, Mol. Phys., 68(3), 1989, 745-758

21. C. B. Moore and J. C. Weisshaar, Formaldehyde Photochemistry, Ann. Rev. Phys. Chem., 34:525555, 1983

22. S. Strickler and R. Barnhart, Absolute Vibronic Intensities in the $A^{1} A_{2}-X^{1} A_{1}$ absorption spectrum of formaldehyde, J. Phys. Chem, 86:448 f., 1983

23. N. Graf, J. Gronki, Ch. Schulz, T. Baritaud, J. Cherel, P. Duret, J. Lavy: In-Cylinder Combustion Visualisation in an Auto-Igniting Gasoline Engine using Fuel-Tracer- and Formaldehyde-LIF imaging, SAE Paper 2001-01-1924, 2001.

\section{CONTACT}

Robert SchießI

Institut für Technische Thermodynamik

Universität Karlsruhe

Kaiserstrasse 12

D-76128 Karlsruhe

schiessl@itt.uni-karlsruhe.de 